\title{
HIRAS: High-resolution IRAS images
}

\author{
Tj. Romke Bontekoe \\ European Space Agency - ESTEC \\ Astrophysics Division \\ Postbus 299, 2200 AG Noordwijk, The Netherlands \\ Do Kester \\ Laboratory for Space Research \\ Postbus 800, 9700 AV Groningen, The Netherlands
}

The InfraRed Astronomical Satellite (IRAS) all-sky survey was designed and optimized for the detection of point sources. This allowed the survey to be conducted in the form of narrow strip scans with redundant coverage of the sky, but with non-uniform covering densities. The data shows in addition to point sources many sources of extended emission, which are best analyzed from images. However, the non-uniform coverage now forms a significant obstacle in the image (re-)construction. Low resolution images, such as in the Infrared Sky Survey Atlas, yield spatial resolutions of 5-10 times the IRAS telescope diffraction limit; HIRAS improves this to 1-2 times!

HIRAS is a Groningen Image Processing SYstem (GIPSY) task which drives the MEMSYS5 algorithm and is specifically designed for IRAS image construction (MEMSYS is a trademark of Maximum Entropy Data Consultants Ltd. Cambridge, UK). In HIRAS the imaging equation

$$
D=R * f \pm \sigma,
$$

is solved. Here $D$ is the data, $f$ is the image, the matrix $R$ describes the instrumental response, and $\sigma$ is the estimated noise in the data. High demands are placed on the quality of the data and the response matrix $R$. Therefore, HIRAS utilizes the individual response functions of the focal plane detectors and rotates them according to the scan angle over the requested map.

The data $D$, still in the form of scans, are first calibrated against a low-resolution map by the IMAGE task in GIPSY. After a first convergence of HIRAS subsequent refinements in the calibration can be performed, now against the new high-resolution map. Corrections in the baseline, drift, and detector gain are applied and continuation of the HIRAS run shows a significant improvement in the result.

In HIRAS the spatial correlations in an image can be controlled interactively. This is done via a new multi-channel method, pyramid images, in which virtually all spatial frequencies are represented. In a pyramid image a $64 \times 64$ pixel image is the sum of a $64 \times 64$, a $32 \times 32, \ldots$, a $2 \times 2$, and a $1 \times 1$ pixel channel, all covering the same map area.

In image reconstruction there is an inevitable amplification of the noise in the data, due to the illconditioned nature of the inversion of the imaging equation. In HIRAS this amplification of the noise can be visualized by the computation of an error map, having identical dimensions to the requested map. The error map represents a full propagation of $\sigma$ through the inversion of the imaging equation. Comparing the image with the error map gives indispensable information about the authenticity of detailed features. For example, the flux of point sources in the IRAS Point Source Catalog is usually within the error as determined by HIRAS. 